\title{
Quality of Chillies (Capsicum annuum L.) as Influenced by Organic Manures Application
}

\author{
P. Malathi* \\ Department of Soil Science and Agricultural Chemistry, Tamil Nadu Agricultural University, \\ Coimbatore-3, India \\ *Corresponding author
}

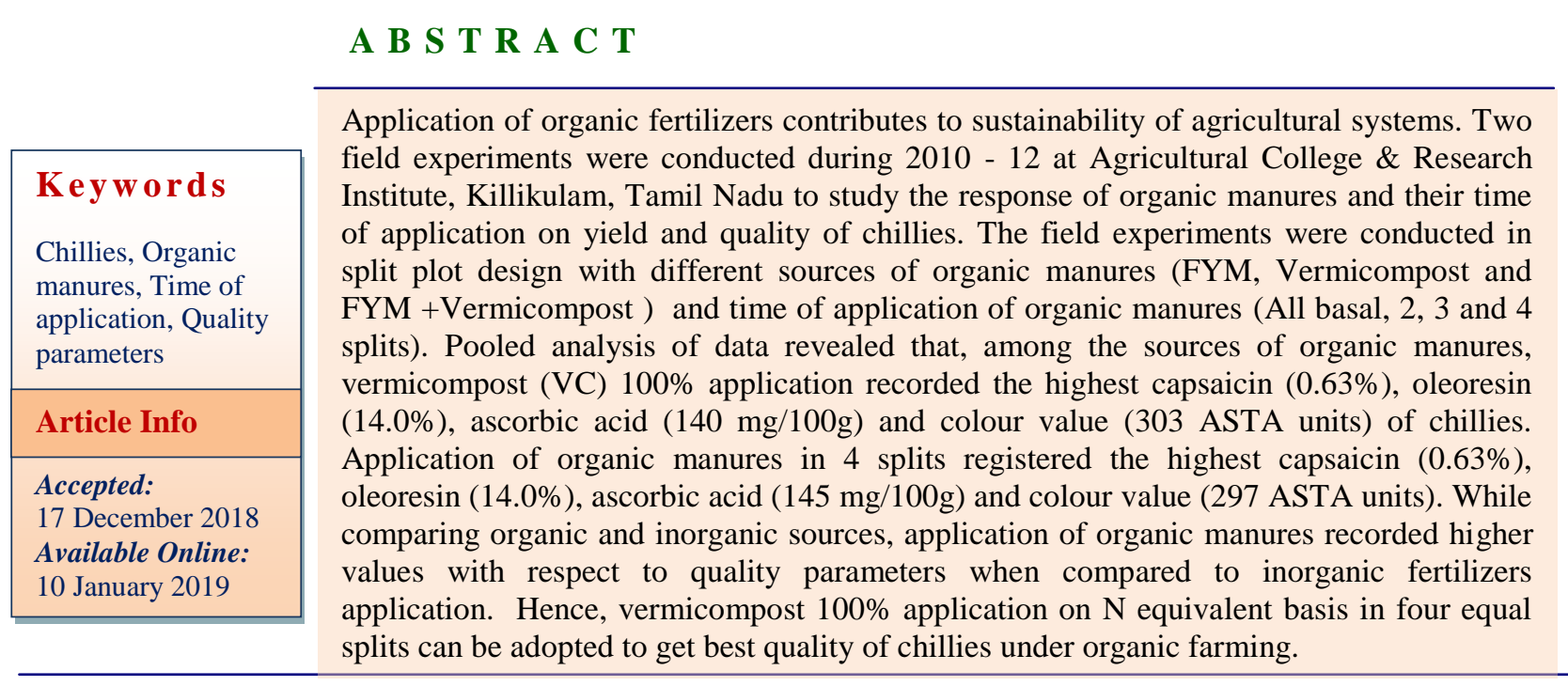

\section{Introduction}

Chillies (Capsicum annnum L.), a member of the family Solanaceae is an important commercial spice cum vegetable crop of India. There is no spice probably so popular as chilli and no other spice has become such an indispensable ingredient of the daily food of majority people of the world. The area under chillies in India is 805 thousand hectares with a production of 1276 thousand tonnes during the year 2011-12 (Anonymous, 2013). Both green and dried chillies are the important components of our routine diet which give the required pungency, colour, taste and flavour to our dishes. The presence of capsaicin in chilli is responsible for its pungency and it has medicinal value. There is lot of demand for chilli oleoresin in the world market. It is having lot of export potential. Chilli is a rich source of vitamin ' $C$ ' and ' $A$ ' with plenty of minerals. The principal colouring matter is capsanthin, the carotenoid pigment which contributes about 35 per cent to the total pigments. 
Consequent upon Green Revolution, the use of high yielding and fertilizer responsive varieties and cultivation system has been intensified and this prompted the use of chemical fertilizers and pesticides. Indiscriminate use of chemical fertilizers and pesticides led to several harmful effects on soil, water and environment causing their pollution and decline in the productivity of the soil. Organic farming helps in rejuvenating the degraded soil and ensure sustainability of crop production. Global awareness of health and environmental issues is increasing in recent years and the demand for organic agricultural products is increasing day by day. Moreover, they are ready to pay a premium price for such foods.

Chilli being a major spice with tremendous export potential, the emphasis needs to be given for increasing the quality apart from productivity and both can be achieved by optimum and balanced supply of all the plant required nutrients. Application of FYM improves quality of chilli by enhancing ascorbic acid content (Chavan et al., 1997), oleoresin content (Malawadi, 2003) and colour value. Jayasree (2006) observed an improvement in quality of chilli due to Panchagavya spray. In this context, it is felt that organic nutrition is a remedy to manage the ill effects of chemical farming so as to manage soil health for sustaining the soil productivity and quality of chilli. In the light of the above facts, the present investigation was conducted to study the effect of organic manures and their time of application on quality of chillies (Capsicum annuum L.) under organic farming.

\section{Materials and Methods}

Two field experiments were conducted during the season Sep-Oct of 2010-11 and 2011-12 at Agricultural College \&Research Institute, Killikulam, Tamil Nadu with chillies var. KKM1 as test crop. The field experiments were conducted in split plot design and replicated thrice. The different sources of organic manures (FYM, Vermicompost and FYM + Vermicompost) were taken in main plot. Time of application of organic manures (All basal, 2, 3 and 4 splits) was taken as subplot treatments. The second field experiment was conducted in the same field and in the same plots as continuous experiment.

The treatments Blanket recommendation $\left(\mathrm{T}_{13}\right)$, STCR - IPNS recommendation $\left(\mathrm{T}_{14}\right)$ and absolute control $\left(\mathrm{T}_{15}\right)$ were raised in adjacent field of same soil type as the inorganic treatments should not be mixed with organic plots. The soil of the experimental field was sandy clay in texture with $\mathrm{pH}$ of 7.45 , EC of $0.26 \mathrm{dSm}^{-1}$ and organic carbon content of $0.52 \%$. The available $\mathrm{N}, \mathrm{P}$ and $\mathrm{K}$ values of the initial soil are 247, 21.8 and $302 \mathrm{~kg} \mathrm{ha}^{-1}$ respectively. For inorganic treatments, the available N, P and $\mathrm{K}$ values of the initial soil are 242, 19.6 and $339 \mathrm{~kg} \mathrm{ha}{ }^{-1}$ respectively for field experiment I and 261, 21.7 and $347 \mathrm{~kg} \mathrm{ha}^{-1}$ respectively for field experiment II.

Based on the initial soil test values, the manures and fertilizers recommendation for STCR-IPNS treatment for a yield target of 25 $\mathrm{q} \mathrm{ha}{ }^{-1}$ was calculated using the following fertilizer prescription equations. The fertilizer doses after adjusting the contributions through FYM@25 $\mathrm{t} \mathrm{ha}^{-1}$ were 60:36:16 kg ha ${ }^{-1}$ $\mathrm{N}: \mathrm{P}_{2} \mathrm{O}_{5}: \mathrm{K}_{2} \mathrm{O}$ and 60:30:15 kg $\mathrm{ha}^{-1}$ $\mathrm{N}: \mathrm{P}_{2} \mathrm{O}_{5}: \mathrm{K}_{2} \mathrm{O}$ respectively for the field experiments I and II. The fertilizer prescription equations followed for STCRIPNS treatments in chillies (red soil) is as follows.

$\mathrm{FN}=8.29 \mathrm{~T}-0.32 \mathrm{SN} ; \mathrm{FP}_{2} \mathrm{O}_{5}=7.13 \mathrm{~T}-$ $5.24 \mathrm{SP} ; \mathrm{FK}_{2} \mathrm{O}=5.86 \mathrm{~T}-0.15 \mathrm{SK}$

Where, FN - Fertilizer N supplied in $\mathrm{kg} \mathrm{ha}^{-1}$; $\mathrm{F}_{2} \mathrm{O}_{5}$ - Fertilizer $\mathrm{P}_{2} \mathrm{O}_{5}$ supplied in $\mathrm{kg} \mathrm{ha}^{-1}$; $\mathrm{FK}_{2} \mathrm{O}$ - Fertilizer $\mathrm{K}_{2} \mathrm{O}$ supplied in $\mathrm{kg} \mathrm{ha}^{-1}$; $\mathrm{T}$ - 
Yield target in Q ha ${ }^{-1} ; \mathrm{SN}$ - Soil available $\mathrm{N}$ in $\mathrm{kg} \mathrm{ha}^{-1}$; SP - Soil available $\mathrm{P}$ in $\mathrm{kg} \mathrm{ha}^{-1}$; SK - Soil available $\mathrm{K}$ in $\mathrm{kg} \mathrm{ha}^{-1}$

Being organic chilli, organic farming practices were followed. The total N, P and K content of FYM and Vermicompost were analysed and the values given in Table 1 . The organic manures were applied on $\mathrm{N}$ equivalent basis $\left(120 \mathrm{~kg} \mathrm{~N} \mathrm{ha}^{-1}\right)$ as basal and on 30, 60 and 90 days after planting as per the treatment schedule. Ascorbic acid content was analyzed in matured fresh green chillies selected at random from each treatment (Sadasivam and Manickam, 2005).

The capsaicin, oleoresin and colour value were determined in the representative composite samples of sundried red chilli fruits collected from each treatment as per the methods suggested by Sadasivam and Manickam (2005). Data from two field experiments and pooled mean were analysed statistically as per Gomez and Gomez (1984). The treatments $T_{13}, T_{14}$ and $T_{15}$ (inorganic treatments) were not included in statistical analysis and the mean values were used for comparison with organic manures application.

\section{Results and Discussion}

The pooled analysis of the data revealed that, among the sources of organic manures, vermicompost (VC) $100 \%$ application recorded the highest capsaicin $(0.63 \%)$, oleoresin (14.0\%), ascorbic acid (140 $\mathrm{mg} / 100 \mathrm{~g}$ ) and colour value (303 ASTA units) followed by FYM 50\% + VC50\% and FYM
$100 \%$ (Tables 2 to 5). Vermicompost performed better than FYM which may be due to the higher nutrient content and also the presence of many plant growth hormones and beneficial microorganisms in vermicompost (Barik and Gulati, 2009).

Shashidhara et al. (2007) reported substantial increase in ascorbic acid content of chilli (Capsicum annuum L.) with the application of organics. Similar increase in ascorbic acid content due to application of organics in chilli was reported by Das and Mishra (1972). Chavan et al. (1997) opined that the increased content of ascorbic acid due to better nutrient availability and nutrient uptake. Nanthakumar and Veeraragavathatham (2001) recorded significantly higher ascorbic acid content over control due to application of crop residues.

Application of organic manures in 4 splits registered the highest capsaicin $(0.63 \%)$, oleoresin (14.0\%), ascorbic acid (145 $\mathrm{mg} / 100 \mathrm{~g}$ ) and colour value (297 ASTA units) followed by 3 split application which were on par (Tables 2 to 5). This might be due to the reason that with increasing number of splits, the nutrients are made available throughout the crop growth period. The study conducted by Ahmad et al. (2007) revealed that the rate of mineralization ( process by which nutrients are released from the manures) of $\mathrm{N}$ enriched compost increases sharply up to 10 days of incubation and then it becomes constant. Higher uptake of nutrients led to enhanced synthesis of volatile oil in seeds and pericarp resulting in enhanced oleoresin yield (Kanner, et al., 1997).

Table.1 Nutrient content of organic manures used in the experiments

\begin{tabular}{|l|c|c|}
\hline Nutrient & FYM & Vermicompost \\
\hline Total N \% & 0.50 & 1.20 \\
\hline Total P \% & 0.16 & 0.61 \\
\hline Total K \% & 0.32 & 0.74 \\
\hline
\end{tabular}


Table.2 Capsaicin (\%) as influenced by sources and time of application of organic manures

\begin{tabular}{|c|c|c|c|c|c|c|c|c|c|c|c|c|}
\hline \multirow[t]{2}{*}{ Treatments } & \multicolumn{4}{|c|}{ Field Experiment I } & \multicolumn{4}{|c|}{ Field Experiment II } & \multicolumn{4}{|c|}{ Pooled mean } \\
\hline & FYM & VC & $\begin{array}{c}\text { FYM + } \\
\text { VC }\end{array}$ & Mean & FYM & VC & $\begin{array}{c}\text { FYM + } \\
\text { VC }\end{array}$ & Mean & FYM & VC & FYM + VC & Mean \\
\hline All basal & 0.51 & 0.58 & 0.56 & 0.55 & 0.53 & 0.61 & 0.59 & 0.58 & 0.52 & 0.59 & 0.58 & 0.56 \\
\hline 2 splits & 0.52 & 0.59 & 0.57 & 0.56 & 0.55 & 0.63 & 0.60 & 0.59 & 0.54 & 0.61 & 0.58 & 0.58 \\
\hline 3 splits & 0.56 & 0.61 & 0.58 & 0.59 & 0.59 & 0.66 & 0.62 & 0.62 & 0.58 & 0.64 & 0.60 & 0.61 \\
\hline 4 splits & 0.58 & 0.66 & 0.60 & 0.61 & 0.61 & 0.70 & 0.63 & 0.65 & 0.59 & 0.68 & 0.61 & 0.63 \\
\hline \multirow[t]{2}{*}{ Mean } & 0.54 & 0.61 & 0.58 & & 0.57 & 0.65 & 0.61 & & 0.56 & 0.63 & 0.60 & \\
\hline & \multicolumn{2}{|c|}{ SEd } & \multicolumn{2}{|c|}{$\mathrm{CD}(\mathrm{P}=0.05)$} & \multicolumn{2}{|c|}{ SEd } & \multicolumn{2}{|c|}{$\mathrm{CD}(\mathrm{P}=0.05)$} & \multicolumn{2}{|c|}{ SEd } & \multicolumn{2}{|c|}{$\mathrm{CD}(\mathrm{P}=\mathbf{0 . 0 5})$} \\
\hline $\mathbf{M}$ & \multicolumn{2}{|c|}{0.01} & \multicolumn{2}{|c|}{0.03} & \multicolumn{2}{|c|}{0.01} & \multicolumn{2}{|c|}{0.04} & \multicolumn{2}{|c|}{0.01} & \multicolumn{2}{|c|}{0.04} \\
\hline $\mathbf{S}$ & \multicolumn{2}{|c|}{0.02} & \multicolumn{2}{|c|}{0.04} & \multicolumn{2}{|c|}{0.02} & \multicolumn{2}{|c|}{0.04} & \multicolumn{2}{|c|}{0.02} & \multicolumn{2}{|c|}{0.04} \\
\hline$M$ at $S$ & \multicolumn{2}{|c|}{0.03} & \multicolumn{2}{|c|}{$\mathrm{NS}$} & \multicolumn{2}{|c|}{0.03} & \multicolumn{2}{|c|}{$\mathrm{NS}$} & \multicolumn{2}{|c|}{0.03} & \multicolumn{2}{|l|}{$\mathrm{NS}$} \\
\hline $\mathbf{S}$ at $\mathbf{M}$ & \multicolumn{2}{|c|}{0.03} & \multicolumn{2}{|c|}{ NS } & \multicolumn{2}{|c|}{0.03} & \multicolumn{2}{|c|}{ NS } & \multicolumn{2}{|c|}{0.03} & \multicolumn{2}{|l|}{ NS } \\
\hline
\end{tabular}

Table.2a Capsaicin (\%) as influenced by inorganic fertilizers

\begin{tabular}{|c|c|c|c|}
\hline Treatments & Field Experiment I & Field Experiment II & Pooled mean \\
\hline Blanket recommendation & 0.53 & 0.54 & 0.54 \\
\hline STCR - IPNS recommendation & 0.56 & 0.57 & 0.57 \\
\hline Absolute control & 0.45 & 0.44 & 0.45 \\
\hline
\end{tabular}


Table.3 Oleoresin (\%) as influenced by sources and time of application of organic manures

\begin{tabular}{|c|c|c|c|c|c|c|c|c|c|c|c|c|}
\hline \multirow[t]{2}{*}{ Treatments } & \multicolumn{4}{|c|}{ Field Experiment I } & \multicolumn{4}{|c|}{ Field Experiment II } & \multicolumn{4}{|c|}{ Pooled mean } \\
\hline & $\begin{array}{c}\text { FY } \\
\text { M }\end{array}$ & $\mathrm{VC}$ & $\begin{array}{c}\text { FYM + } \\
\text { VC }\end{array}$ & Mean & $\begin{array}{l}\text { FY } \\
\text { M }\end{array}$ & $\mathrm{VC}$ & $\begin{array}{c}\text { FYM + } \\
\text { VC }\end{array}$ & Mean & $\begin{array}{c}\text { FY } \\
\mathbf{M}\end{array}$ & $\mathbf{V C}$ & FYM + VC & Mean \\
\hline All basal & 12.1 & 13.2 & 12.5 & 12.6 & 12.4 & 13.6 & 12.7 & 12.9 & 12.3 & 13.4 & 12.6 & 12.8 \\
\hline 2 splits & 12.2 & 13.9 & 13.1 & 13.1 & 12.5 & 14.2 & 13.5 & 13.4 & 12.4 & 14.0 & 13.3 & 13.2 \\
\hline 3 splits & 13.1 & 14.1 & 13.5 & 13.6 & 13.5 & 14.5 & 13.9 & 14.0 & 13.3 & 14.3 & 13.7 & 13.8 \\
\hline 4 splits & 13.3 & 14.2 & 13.9 & 13.8 & 13.7 & 14.6 & 14.2 & 14.2 & 13.5 & 14.4 & 14.1 & 14.0 \\
\hline \multirow[t]{2}{*}{ Mean } & 12.7 & 13.9 & 13.2 & & 13.0 & 14.2 & 13.6 & & 12.8 & 14.0 & 13.4 & \\
\hline & \multicolumn{2}{|c|}{ SEd } & \multicolumn{2}{|c|}{$\mathrm{CD}(\mathrm{P}=0.05)$} & \multicolumn{2}{|c|}{ SEd } & \multicolumn{2}{|c|}{$\mathrm{CD}(\mathrm{P}=0.05)$} & \multicolumn{2}{|c|}{ SEd } & \multicolumn{2}{|c|}{$\mathrm{CD}(\mathrm{P}=0.05)$} \\
\hline $\mathbf{M}$ & \multicolumn{2}{|c|}{0.3} & \multicolumn{2}{|c|}{0.8} & \multicolumn{2}{|c|}{0.3} & \multicolumn{2}{|c|}{0.8} & \multicolumn{2}{|c|}{0.3} & \multicolumn{2}{|c|}{0.8} \\
\hline $\mathbf{S}$ & \multicolumn{2}{|c|}{0.4} & \multicolumn{2}{|c|}{ NS } & \multicolumn{2}{|c|}{0.5} & \multicolumn{2}{|c|}{1.0} & \multicolumn{2}{|c|}{0.5} & \multicolumn{2}{|l|}{0.9} \\
\hline$M$ at $S$ & \multicolumn{2}{|c|}{0.7} & \multicolumn{2}{|c|}{ NS } & \multicolumn{2}{|c|}{0.7} & \multicolumn{2}{|c|}{ NS } & \multicolumn{2}{|c|}{0.7} & \multicolumn{2}{|l|}{ NS } \\
\hline$S$ at $M$ & \multicolumn{2}{|c|}{0.7} & \multicolumn{2}{|c|}{ NS } & \multicolumn{2}{|c|}{0.8} & \multicolumn{2}{|c|}{ NS } & \multicolumn{2}{|c|}{0.8} & \multicolumn{2}{|l|}{ NS } \\
\hline
\end{tabular}

Table.3a Oleoresin (\%) as influenced by inorganic fertilizers

\begin{tabular}{|c|c|c|c|}
\hline Treatments & Field Experiment I & Field Experiment II & Pooled mean \\
\hline Blanket recommendation & 13.1 & 13.2 & 13.2 \\
\hline STCR - IPNS recommendation & 13.4 & 13.5 & 13.5 \\
\hline Absolute control & 12.2 & 12.3 & 12.2 \\
\hline
\end{tabular}


Table.4 Ascorbic acid (mg/100g) as influenced by sources and time of application of organic manures

\begin{tabular}{|c|c|c|c|c|c|c|c|c|c|c|c|c|}
\hline \multirow[t]{2}{*}{ Treatments } & \multicolumn{4}{|c|}{ Field Experiment I } & \multicolumn{4}{|c|}{ Field Experiment II } & \multicolumn{4}{|c|}{ Pooled mean } \\
\hline & FYM & VC & $\begin{array}{c}\text { FYM + } \\
\text { VC }\end{array}$ & Mean & $\begin{array}{l}\text { FY } \\
\text { M }\end{array}$ & VC & $\begin{array}{c}\text { FYM + } \\
\text { VC }\end{array}$ & Mean & $\begin{array}{l}\text { FY } \\
\text { M }\end{array}$ & VC & FYM + VC & Mean \\
\hline All basal & 112 & 121 & 117 & 117 & 119 & 129 & 124 & 124 & 116 & 125 & 121 & 120 \\
\hline 2 splits & 119 & 125 & 122 & 122 & 125 & 135 & 131 & 130 & 122 & 130 & 127 & 126 \\
\hline 3 splits & 133 & 139 & 135 & 136 & 139 & 148 & 146 & 144 & 136 & 144 & 141 & 140 \\
\hline 4 splits & 123 & 158 & 142 & 141 & 131 & 167 & 151 & 150 & 127 & 163 & 147 & 145 \\
\hline \multirow[t]{2}{*}{ Mean } & 122 & 136 & 129 & & 129 & 145 & 138 & & 125 & 140 & 134 & \\
\hline & \multicolumn{2}{|c|}{ SEd } & \multicolumn{2}{|c|}{$\mathrm{CD}(\mathrm{P}=0.05)$} & \multicolumn{2}{|c|}{ SEd } & \multicolumn{2}{|c|}{$\mathrm{CD}(\mathrm{P}=0.05)$} & \multicolumn{2}{|c|}{ SEd } & \multicolumn{2}{|c|}{$\mathrm{CD}(\mathrm{P}=0.05)$} \\
\hline $\mathbf{M}$ & \multicolumn{2}{|c|}{3} & \multicolumn{2}{|c|}{8} & \multicolumn{2}{|c|}{3} & \multicolumn{2}{|c|}{9} & \multicolumn{2}{|c|}{3} & \multicolumn{2}{|c|}{8} \\
\hline $\mathbf{S}$ & \multicolumn{2}{|c|}{4} & \multicolumn{2}{|c|}{9} & \multicolumn{2}{|c|}{5} & \multicolumn{2}{|c|}{10} & \multicolumn{2}{|c|}{5} & \multicolumn{2}{|l|}{10} \\
\hline$M$ at $S$ & \multicolumn{2}{|c|}{7} & \multicolumn{2}{|c|}{ NS } & \multicolumn{2}{|c|}{8} & \multicolumn{2}{|c|}{ NS } & \multicolumn{2}{|c|}{7} & \multicolumn{2}{|l|}{ NS } \\
\hline $\mathbf{S}$ at $\mathbf{M}$ & \multicolumn{2}{|c|}{8} & \multicolumn{2}{|c|}{ NS } & \multicolumn{2}{|c|}{7} & \multicolumn{2}{|c|}{ NS } & \multicolumn{2}{|c|}{8} & \multicolumn{2}{|l|}{ NS } \\
\hline
\end{tabular}

Table.4a Ascorbic acid (mg/100g) as influenced by inorganic fertilizers

\begin{tabular}{|c|c|c|c|}
\hline Treatments & Field Experiment I & Field Experiment II & Pooled mean \\
\hline Blanket recommendation & 115 & 121 & 118 \\
\hline STCR - IPNS recommendation & 121 & 132 & 127 \\
\hline Absolute control & 100 & 103 & 102 \\
\hline
\end{tabular}


Table.5 Colour Value (ASTA units) as influenced by sources and time of application of organic manures

\begin{tabular}{|c|c|c|c|c|c|c|c|c|c|c|c|c|}
\hline \multirow[t]{2}{*}{ Treatments } & \multicolumn{4}{|c|}{ Field Experiment I } & \multicolumn{4}{|c|}{ Field Experiment II } & \multicolumn{4}{|c|}{ Pooled mean } \\
\hline & FYM & VC & $\begin{array}{c}\text { FYM + } \\
\text { VC }\end{array}$ & Mean & FYM & VC & $\begin{array}{c}\text { FYM + } \\
\text { VC }\end{array}$ & Mean & FYM & VC & FYM + VC & Mean \\
\hline All basal & 243 & 286 & 265 & 265 & 249 & 294 & 271 & 271 & 246 & 290 & 268 & 268 \\
\hline 2 splits & 255 & 295 & 277 & 276 & 262 & 304 & 284 & 283 & 259 & 300 & 281 & 280 \\
\hline 3 splits & 263 & 302 & 288 & 284 & 271 & 312 & 293 & 292 & 267 & 307 & 291 & 288 \\
\hline 4 splits & 278 & 308 & 293 & 293 & 285 & 319 & 301 & 302 & 282 & 314 & 297 & 297 \\
\hline \multirow[t]{2}{*}{ Mean } & 260 & 298 & 281 & & 267 & 307 & 287 & & 263 & 303 & 284 & \\
\hline & \multicolumn{2}{|c|}{ SEd } & \multicolumn{2}{|c|}{$\mathrm{CD}(\mathrm{P}=0.05)$} & \multicolumn{2}{|c|}{ SEd } & \multicolumn{2}{|c|}{$\mathrm{CD}(\mathrm{P}=0.05)$} & \multicolumn{2}{|c|}{ SEd } & \multicolumn{2}{|c|}{$\mathrm{CD}(\mathrm{P}=0.05)$} \\
\hline $\mathbf{M}$ & \multicolumn{2}{|c|}{6} & \multicolumn{2}{|c|}{16} & \multicolumn{2}{|c|}{6} & \multicolumn{2}{|c|}{17} & \multicolumn{2}{|c|}{6} & \multicolumn{2}{|c|}{17} \\
\hline $\mathbf{S}$ & \multicolumn{2}{|c|}{9} & \multicolumn{2}{|c|}{20} & \multicolumn{2}{|c|}{10} & \multicolumn{2}{|c|}{20} & \multicolumn{2}{|c|}{9} & \multicolumn{2}{|l|}{20} \\
\hline $\mathbf{M}$ at $\mathbf{S}$ & \multicolumn{2}{|c|}{15} & \multicolumn{2}{|c|}{ NS } & \multicolumn{2}{|c|}{16} & \multicolumn{2}{|c|}{ NS } & \multicolumn{2}{|c|}{15} & \multicolumn{2}{|l|}{$\mathrm{NS}$} \\
\hline $\mathbf{S}$ at $\mathbf{M}$ & \multicolumn{2}{|c|}{16} & \multicolumn{2}{|c|}{ NS } & \multicolumn{2}{|c|}{15} & \multicolumn{2}{|c|}{ NS } & \multicolumn{2}{|c|}{16} & \multicolumn{2}{|l|}{ NS } \\
\hline
\end{tabular}

Table.5a Colour Value (ASTA units) as influenced by inorganic fertilizers

\begin{tabular}{|c|c|c|c|}
\hline Treatments & Field Experiment I & Field Experiment II & Pooled mean \\
\hline Blanket recommendation & 249 & 255 & 252 \\
\hline STCR - IPNS recommendation & 265 & 274 & 270 \\
\hline Absolute control & 223 & 227 & 225 \\
\hline
\end{tabular}


Application of organic manures recorded higher values with respect to quality parameters when compared to inorganic fertilizers application (Tables 2a to 5a). While comparing organic and inorganic sources, quality of chillies (capsaicin $0.57 \%$, oleoresin $-13.5 \%$, ascorbic acid - 127 $\mathrm{mg} / 100 \mathrm{~g}$ and colour value - 270 ASTA units with inorganics application) was higher with organic manures application. Shashidhara et al. (2007) observed increased oleoresin content of chillies with the application of organic manures when compared to the application of recommended dose of fertilizers. Vijayakumari et al., (2007) reported enhanced carotenoid and $\beta$-carotene content with the application of FYM over inorganic fertilizers application. Hence, vermicompost $100 \%$ application on $\mathrm{N}$ equivalent basis in four equal splits can be adopted to get higher quality of chillies under organic farming.

\section{References}

Ahmad, R., Arshad, M., Naveed, M., Zahir, Z. A., Sultan, T. and Khalia, M. (2007). Carbon mineralization rate of composted and raw organic wastes and its implications on environment. Soil \& Environ. 26(1): 92-96.

Anonymous. (2013). State of Indian Agriculture 2012-13, Government of India, Ministry of Agirculture, Department of Agriculture and Co-operation, New Delhi. pp. 200-206.

Barik, A. K., Das, A., Giri, A. K. and Chattopadhyay, G. N. (2006). Effect of organic (vermicompost, farm yard manure) and chemical sources of plant nutrients on productivity and soil fertility of kharif rice (Oryza sativa L.). Crop research 31(3): 338-342.

Chavan, P.J., Jimail, S., Rudrakha, G.b., Malewar, G.V. and Baig, M.I., 1997, Effect of various nitrogen levels through FYM and urea on yield and uptake of nutrients and ascorbic acid content of chilli. J. Indian Soc. Soil Sci., 45: 833-835.
Das, R.C. and Mishra, S.N. 1972. Effect of nitrogen, phosphorus and potassium on growth, yield and quality of chilli (Capsicum annuum L.). Plant Science, 4 : 78-83.

Gomez, K. A. and Gomez, A. A. (1984). Statistical procedures for agricultural research. Second Edition. A Wiley Inter Science Publication (John Wiley and Sons), New York. pp. 20-30.

Jayasree, P. and Annamma, G., 2006, Do biodynamic practices influence yield, quality and economics of cultivation of chilli (Capsicum annuum L.)? J. Tropical Agric., 44(1-2): 68-70.

Kanner, J., Stella, Harel Palevitch, D. and BenGera, I. 1997. Colour retention in sweet red paprika (Capsicum annuum L.) powder as affected by moisture content and ripening stage. J. Food Tech., 12: 59-64.

Malawadi, M.N., Palled, Y.B., Basavaraj, B. and Hiremath, S.M., 2003, Effect of secondary and micronutrients on yield and quality of chilli (Capsicum annuum L.). M.Sc.(Agri.) Thesis, Univ. Agric. Dharwad (India).

Nanthakumar and Veeraragavathatham. 2001. Effect of integrated nutrient management on yield and quality attributes of brinjal (Solanum melongena cv. Pirl). South Indian Hort., 49: 195-198.

Sadasivam, S. and A. Manickam. 2005. Biochemical Methods. New Age International (P) Ltd.

Shashidhara, G. B., Thimma Naik, M., Bidari, B. I. and Yenagi, B. S. (2007). Studies on the effect of organic manures on growth, yield and quality of chilli (Capsicum annuum L.) under black soils of northern transition zone of Karnataka. Indian J. Crop Science 2(2) : 315-317.

Vijayakumari, B., Hiranmai, R. and Sowmya, M. 2007. Organic manures on the yield of carrot. Kisan World: 61-62.

\section{How to cite this article:}

Malathi, P. 2019. Quality of Chillies (Capsicum annuum L.) as Influenced by Organic Manures Application. Int.J.Curr.Microbiol.App.Sci. 8(01): 2811-2818. doi: https://doi.org/10.20546/ijcmas.2019.801.295 